\title{
Minireview
}

\section{Nursing some sense out of Myc}

\author{
Nicole M Sodir and Gerard I Evan
}

Address: Department of Pathology and Helen Diller Family Comprehensive Cancer Center, University of California, San Francisco, CA 94143-0502, USA.

Correspondence: Gerard I Evan. Email: gerard.evan@ucsf.edu

\begin{abstract}
Data recently published in $B M C$ Biology provide insights into the normal physiological function of c-myc in the development and regeneration of the mammary gland and indicate a key role in epithelial cell proliferation, elaboration of ductal alveoli, and the biosynthetic capacity and milk production of the mature organ.
\end{abstract}

See related research article $h$ ttp://www.biomedcentral.com/1741-7007/7/63

The three Myc proteins, c-Myc, L-Myc and N-Myc, are all basic helix-loop-helix (bHLH)-Zip transcription factors that coordinate cell proliferation, cell cycle progression, cell growth, metabolism, differentiation and tissue remodeling, as well as a variety of protective checkpoint mechanisms such as growth arrest and apoptosis. Myc proteins form heterodimers with their bHLH-Zip partner Max, transactivating genes by binding at canonical E-Box $\mathrm{CAC}^{\mathrm{G}} / \mathrm{A}_{\mathrm{TG}}$ recognition elements in target gene promoters and repressing other genes through interactions with the zinc finger protein Miz-1 and/or recruitment of the DNA methyltransferase corepressor Dnmt3a (Figure 1) [1]. Expression array, SAGE, chromatin IP, promoter scanning and whole cell proteomic approaches identify thousands of Myc target genes with diverse roles in virtually every aspect of cell and tissue behavior, including growth, metabolism, cell cycle, differentiation, telomere maintenance, DNA damage and repair, intracellular membrane transport, cytoskeleton, cytokine production, angiogenesis, invasion and apoptosis [2] (see also http://www.myc-cancer-gene.org/ site/mycTargetDB.asp).

In normal cells expression of Myc proteins is tightly regulated, either by developmental cues or, in the case of c-Myc expression in adult regenerative tissues, by mitogenic stimulation. In the absence of such proactive induction, short-lived Myc mRNAs and proteins are rapidly cleared from cells, which then default back to their non-proliferative state. This tight control of Myc expression is defective in almost all cancers, either because of deregulating mutations in the Myc genes themselves, or through the relentless induction of Myc expression by upstream oncogenic signals such as the Wnt/ $\beta$-catenin, Notch or RTK/Ras pathways. Moreover, ectopic activation of Myc is sufficient in many adult tissues to engage, coordinate and maintain the diverse intracellular (cell growth, cell cycle progression, biosynthetic metabolism, ribogenesis and translation) and extracellular (release of cytokines and chemokines, recruitment of inflammatory cells, extensive stromal remodeling, invasion and angiogenesis) processes that somatic cells require for their orderly expansion (Figure 1). Myc's highly pleiotropic effects are mirrored by its wide range of gene targets, estimated to encompass some $20 \%$ of all vertebrate genes [2].

It is notable that the role of Myc as a pivotal coordinator of cell proliferation is common to almost all adult tissues. Since different tissues vary widely in their architecture, regenerative capacity and the risks of infection and oncogenesis, many of Myc's potential target genes are likely to be regulated in a context-dependent fashion; that is, the extent of their control by Myc (and, hence, the actual execution of their cognate biological programs) is dependent on other factors such as cell type and history, local environment and circumstance.

\section{Effects of Myc inhibition/knockout on normal tissues and cells}

A pressing question in Myc biology is whether or not Myc function is essential for somatic cell proliferation. Myc proteins exert very broad but relatively subtle effects on cells. Their impact on expression of individual genes is generally modest and there is no known gene whose expression is dependent solely on Myc: rather, Myc appears to modify the efficiency with which more bespoke transcription factors regulate their targets, in part through its general impact on chromatin architecture and accessibility [3]. Myc is therefore unlikely to be essential for any single transcriptional program but may nonetheless act as the essential coordinator that integrates all the disparate transcriptional growth programs into a coherent strategic whole. Unfortunately, the experimental data remain unclear on the requirement for Myc function in cell proliferation, one complication being functional complementation between different members of the Myc family, principally c- and N-Myc since L-Myc has much weaker intrinsic transcriptional activity [4]. These may be constitutively 


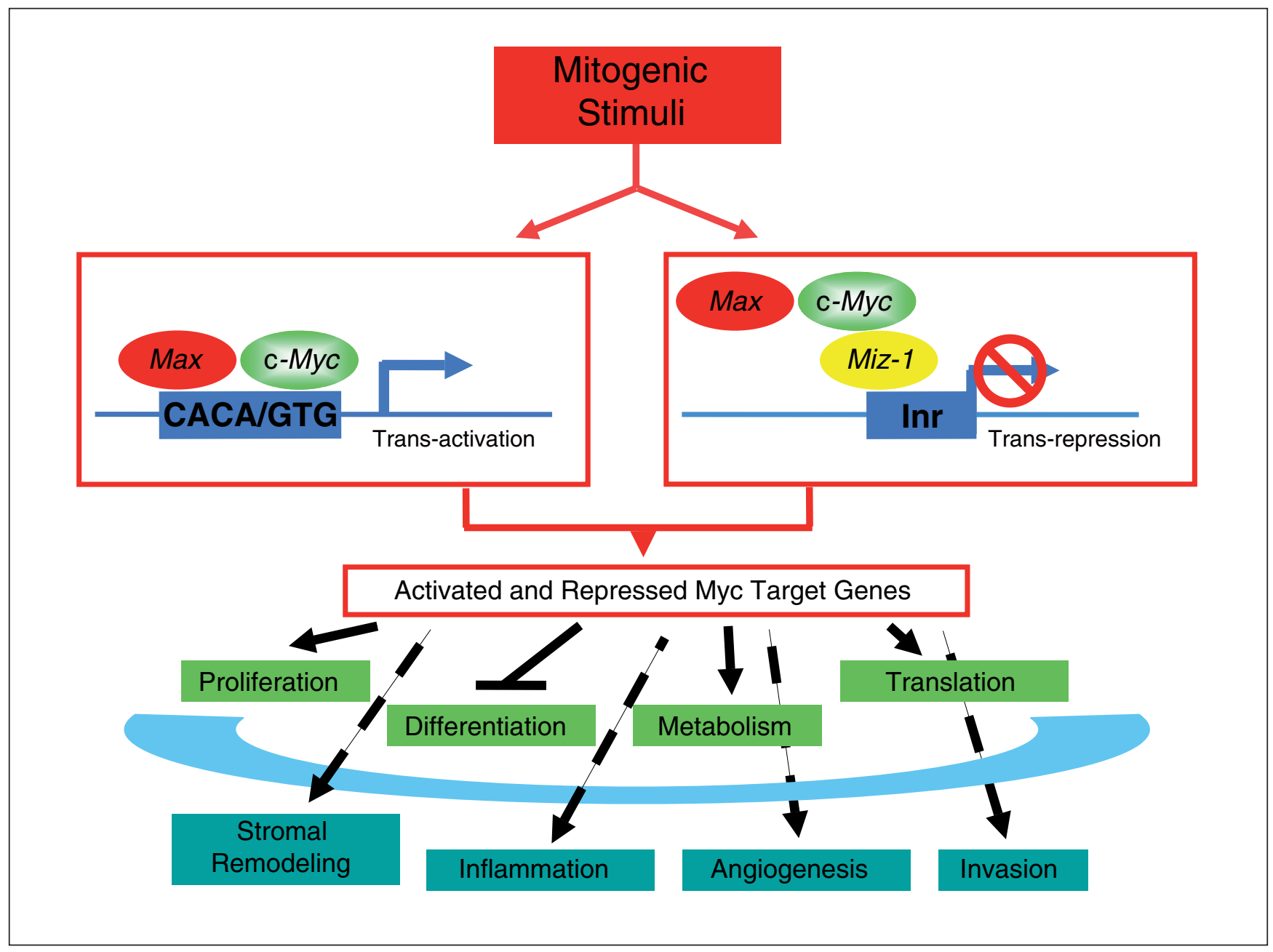

Figure 1

Pleiotropic effects of c-Myc activation. Myc acts as an intracellular sensor of mitogenic stimuli; its expression leads to the activation or repression of an enormous range of target genes that affect diverse intracellular and extracellular biological processes.

co-expressed or induced to compensate for any deficit in their siblings. c-myc-deficient mice die by embryonic day 10.5 (E10.5) with overt hematopoietic, vascular and placental defects [5]. While this indicates a critical and non-redundant role for c-myc in developmental angiogenesis and erythropoiesis, the consequent embryonic lethality obscures any obligate role c-myc might have in tissues beyond this developmental stage. However, it is certain that c-myc is also required for proliferation and elaboration of many other somatic tissues (see below). Germ-line knockouts of $\mathrm{N}-m y c$ in mice also die in utero around E11.5 from profound defects in multiple tissues, including the central and peripheral nervous systems and multiple epithelial tissues [6]. L-Myc-deficient mice, by contrast, show no overt phenotype [7]. Rat1 fibroblast cells do not detectably express L-Myc and N-Myc and, when somatically deleted for c-myc, remain viable but exhibit dramatically slower proliferative rates and severe cell cycle defects. Loss of c-myc compromises multiple phases of the cell cycle, slowing progression through both G1 and G2 and depressing and delaying activities of all cyclin $\mathrm{Cdk}$ complexes. A similar failure to proliferate is also evident in primary mouse embryo fibroblasts derived from mice in which c-myc has been conditionally deleted.

More recently, both conditional deletion and dominant negative inhibition have been used to investigate in vivo the roles of Myc proteins in adult tissues. Surprisingly, initial studies in skin [8] and gut [9] suggested that deletion of c-myc had little impact on maintenance of either regenerative tissue or hematopoietic stem cells [10]. In the latter case at least, such independence from c-Myc is due to co-expression of N-Myc, consistent with known degeneracy in c-Myc and N-Myc function. More recent conditional 
deletion studies in the gastrointestinal (GI) tract, however, suggest that the apparent lack of requirement for c-Myc in tissue homeostasis may be an artifact of relatively low penetrance of expression of the Cre recombinase that excises the c-myc gene, since driving the Cre expression from a more powerful and pervasive promoter induces profound and lethal attrition of intestinal crypts [11]. In addition, systemic expression in vivo of a dominant interfering Myc bHLHL-Zip dimerization domain mutant (Omomyc) that efficiently blocks c-Myc, N-Myc and L-Myc-dependent transcriptional activation triggers a marked reduction of proliferation in tissues with rapid turnover (for example intestinal epithelium, skin, bone marrow, testis) but has no discernible acute effect on adult organs with low proliferative indices (for example pancreas, kidney, liver, heart or lung). Strikingly, this massive reduction in proliferation rate of regenerative tissues in such Omomyc-expressing animals is not accompanied by any cell death or loss of tissue integrity: consequently, such systemic Myc inhibition has no pathological negative impact on the animals, even over extended periods (4 to 12 weeks). Moreover, the phenotypic effects of such Myc inhibition are completely and rapidly reversible upon subsequent Omomyc downregulation [12].

In a recent study published in BMC Biology, Stoelzle et al. [13] investigate the specific physiological role of c-myc in the mammary gland by conditional gene deletion using Cre-loxP recombinase technology. Cre (Cyclization Recombination) is a sequence-specific DNA recombinase that is used to excise stretches of DNA flanked either side by loxP (Locus of X-over P1) DNA sequences. Expression of Cre under the control of a tissue-specific promoter can then be used to delete specific gene sequences in a tissuespecific manner. Whey acidic protein (WAP)iCre transgenic mice were used to delete the loxP-flanked c-myc locus specifically in luminal alveolar cells, starting at midpregnancy and continuing throughout lactation. Affected $c$-mycfl/fl; WAPiCre ${ }^{+}$mothers exhibited a marked deficit in milk production and capacity to nurse their young, associated with a failure of terminal end buds to elaborate alveoli within the lactating mammary gland. Since the $W A P$ promoter is triggered during first pregnancy, parous $c-m y c^{f l / f l} ; W_{A P i C r e}{ }^{+}$start subsequent pregnancies with c-myc already deleted. During such pregnancies, mice show dramatic deficits in mammary proliferation and maturation, although the resulting hypoplastic tissues differentiate normally. This proliferative deficit is mirrored by the markedly reduced efficiency of mammary tissue regeneration when parous $c-m y c^{f l / f l}$; WAPiCre ${ }^{+}$mammary tissue was transplanted into cleared fat pads of immunocompromised recipient mice.

While such data suggest that proliferation of mammary epithelial progenitor cells is still possible in the absence of c-myc, albeit at reduced efficiency, the usual uncertainties of Cre-loxP technology obtain. The progressive appearance of the signature restriction fragment induced by Cre deletion of c-myc clearly indicates accumulation of cells with deleted c-Myc, but it is less clear what proportion of cells retain either one or both functional c-myc allele(s). No surrogate marker of recombination was used to estimate deletion efficiency and, as the authors point out, c-Myc immunohistochemistry remains a capricious and insensitive arbiter of recombination efficiency. Any remaining c-myc-competent cells would be expected to contribute variably to initial growth and subsequent regeneration of mammary tissues and their uneven representation might explain the partial capacity of parous $c-m y c^{f l / f l} ; W A P i C r e^{+}$ mammary tissue to reconstitute the gland in naïve recipients, albeit at reduced efficacy.

It is notable that c-myc-deleted mutant glands exhibit alveolar cells with condensed ER and secretory capacity and reduced levels of ribosomal RNA and milk proteins. They are also far less efficient at producing the principal milk proteins $\alpha$-lactalbumin and $\beta$-casein. Taken together, this indicates an important role for c-myc in establishing, and perhaps maintaining, biosynthetic output of the mammary alveolar cells. Such data recall c-myc deletion in intestinal epithelium, which generates $\mathrm{c}$-Myc-deficient crypt progenitor cells that are not only smaller, cycle more slowly and enter mitosis with decreased cell size, but also exhibit reduced biosynthetic activity relative to their wildtype counterparts. Thus, studies in both mammary and intestinal epithelium concur that c-Myc is important for cells to establish the necessary biosynthetic capacity to progress expeditiously through the cell cycle. However, whether this indicates that c-Myc also plays a role in maintaining the specialized protein synthetic capacity of differentiated mammary epithelial cells is unclear: it is equally plausible that absence of c-Myc during the ontogeny of these secretory cells left them with irremediable structural deficits in intracellular architecture that preclude efficient production of milk proteins. Indeed, this latter notion would be most consistent with the selective deficiency such cells show in production of milk proteins, which presumably requires the unusual secretory architecture of mature mammary alveolar cells but not other 'housekeeping' polypeptides like $\beta$-actin and GAPDH, which do not. Distinguishing between the two possibilities awaits studies in which deleted Myc function is subsequently restored in established mammary epithelium to ascertain whether or not this reinstates biosynthetic capacity, either immediately or during subsequent pregnancy-induced re-growth.

\section{Myc as a target in cancer therapy}

The pivotal, and largely non-redundant, role Myc appears to play in coordinating proliferation of tumor cells has reignited interest in it as a possible target for cancer 
therapy. This notion is strengthened by the widespread deregulation of Myc observed in human cancers and by emerging evidence that inactivation of Myc function can suppress both initiation and evolution of cancers in multiple tissue types and trigger the rapid and complete regression of established tumors, even in cancers where Myc itself is not the 'driving' oncogene [12]. However, since Myc is also required for the proliferation of many normal tissues, there have always been concerns that Myc inhibition might be accompanied by catastrophic side effects in normal tissues. Recently, however, such concerns have been largely allayed by data indicating that the impact of systemic Myc inhibition, at least over the relatively short term, is only mild and completely reversible. The studies by Stoelzle et al. [13] offer additional reassurance by demonstrating that inhibition of Myc is not toxic per se for established, adult mammary tissue, although clearly care would need to be taken in administering any Myc inhibitor during periods of mammary tissue regeneration since this might lead to transient, and perhaps permanent, mammary gland dysfunction. For the moment, pharmacological inhibitors remain a pipe dream. However, advances in drug design, strategy and delivery may some day allow us to target the non-redundant processes that really make tumors tick. Of these, Myc is an obvious and provocative candidate.

\section{References}

1. Adhikary S, Eilers M: Transcriptional regulation and transformation by Myc proteins. Nat Rev 2005, 6:635-645.

2. Cole MD, Henriksson M: $\mathbf{2 5}$ years of the c-Myc oncogene. Semin Cancer Biol 2006, 16:241.

3. Knoepfler PS, Zhang XY, Cheng PF, Gafken PR, McMahon SB, Eisenman RN: Myc influences global chromatin structure. Embo J 2006, 25:2723-2734.

4. Malynn BA, de Alboran IM, O'Hagan RC, Bronson R, Davidson $\mathrm{L}$, DePinho RA, Alt FW: N-myc can functionally replace c-myc in murine development, cellular growth, and differentiation. Genes Dev 2000, 14:1390-1399.
5. Baudino TA, McKay C, Pendeville-Samain H, Nilsson JA, Maclean KH, White EL, Davis AC, Ihle JN, Cleveland JL: c-Myc is essential for vasculogenesis and angiogenesis during development and tumor progression. Genes Dev 2002, 16:2530-2543.

6. Stanton BR, Perkins AS, Tessarollo L, Sassoon DA, Parada LF: Loss of $n$-myc function results in embryonic lethality and failure of the epithelial component of the embryo to develop. Genes Dev 1992, 6:2235-2247.

7. Hatton KS, Mahon K, Chin L, Chiu FC, Lee HW, Peng D, Morgenbesser SD, Horner J, DePinho RA: Expression and activity of L-Myc in normal mouse development. Mol Cell Biol 1996, 16:1794-1804.

8. Oskarsson T, Essers MA, Dubois N, Offner S, Dubey C, Roger C, Metzger D, Chambon P, Hummler E, Beard P, Trumpp A: Skin epidermis lacking the c-Myc gene is resistant to Rasdriven tumorigenesis but can reacquire sensitivity upon additional loss of the p21Cip1 gene. Genes Dev 2006, 20:2024-2029.

9. Bettess MD, Dubois N, Murphy MJ, Dubey C, Roger C, Robine $S$, Trumpp A: c-Myc is required for the formation of intestinal crypts but dispensable for homeostasis of the adult intestinal epithelium. Mol Cell Biol 2005, 25:7868-7878.

10. Wilson A, Murphy MJ, Oskarsson T, Kaloulis K, Bettess MD, Oser GM, Pasche AC, Knabenhans C, Macdonald HR, Trumpp A: c-Myc controls the balance between hematopoietic stem cell self-renewal and differentiation. Genes Dev 2004, 18: 2747-2763.

11. Muncan V, Sansom OJ, Tertoolen L, Phesse TJ, Begthel H, Sancho E, Cole AM, Gregorieff A, de Alboran IM, Clevers H, Clarke AR: Rapid loss of intestinal crypts upon conditional deletion of the Wnt/Tcf-4 target gene c-Myc. Mol Cell Biol 2006, 26:8418-8426.

12. Soucek L, Whitfield J, Martins CP, Finch AJ, Murphy DJ, Sodir NM, Karnezis AN, Swigart LB, Nasi S, Evan GI: Modelling Myc inhibition as a cancer therapy. Nature 2008, 455:679683.

13. Stoelzle T, Scharb P, Trumpp A, Hynes NE: c-Myc affects mRNA translation, cell proliferation and progenitor cell function in the mammary gland. BMC Biol 2009, 7:63.

Published: 28 September 2009

doi:10.1186/jbiol181

(C) 2009 BioMed Central Ltd 